01

\title{
Оптимизация режимов термоэлектрического охлаждения теплонагруженных элементов с учетом термического сопротивления теплоотводящей системы
}

\author{
(C) Е.Н. Васильев
}

Институт вычислительного моделирования СО РАН, ФИЦ „Красноярский научный центр СО РАН“, 660036 Красноярск, Россия e-mail: ven@icm.krasn.ru

\section{(Поступило в Редакцию 9 ноября 2016 г.)}

Представлена математическая модель для расчета и оптимизации режимов термоэлектрического охлаждения теплонагруженных элементов технических и электронных устройств. Модель, основанная на аналитических соотношениях, использует в качестве исходных данных рабочие характеристики термоэлектрических модулей и позволяет с учетом термического сопротивления теплоотводящей системы и тепловой нагрузки определять температурный режим и оптимальные значения силы тока электропитания модулей.

DOI: 10.21883/JTF.2017.09.44899.2094

\section{Введение}

Современные технологии позволяют производить устройства, сочетающие малые размеры и высокое энергопотребление, что обостряет проблему обеспечения оптимальных тепловых режимов, которые соответствуют сравнительно узкому температурному диапазону. Традиционные теплоотводящие системы (ТОC) на основе свободно конвективного теплопереноса или принудительного вентилирования не всегда справляются с задачей охлаждения теплонагруженных элементов (ТНЭ). Перспективным направлением для повышения интенсивности охлаждения ТНЭ и точности терморегулирования является применение термоэлектрических модулей (ТЭМ), которые наделяют ТОС функцией охлаждения.

В работах по термоэлектрическому охлаждению недостаточное внимание уделяется оптимизации режимов ТЭМ с детальным исследованием влияния ТОС на характеристики всей системы охлаждения $[1,2]$. Целью настоящей работы является создание аналитической модели для расчета и оптимизации режимов термоэлектрического охлаждения, позволяющей проводить согласование термического сопротивления ТОС с величиной тепловой нагрузки и силой тока питания ТЭМ для достижения максимальной эффективности термоэлектрической системы охлаждения и терморегулирования (ТЭСОТ) в целом. Использование математической модели, основанной на аналитических соотношениях, существенно расширяет возможности анализа режимов термоэлектрического охлаждения.

Задача оптимизации режима работы ТЭМ сводится к определению оптимальной силы тока, при которой эффективность охлаждения является максимальной. Стандартной характеристикой эффективности термоэлектрического охлаждения является коэффициент СОР (coefficient of performance), равный отношению холодопроизводительности ТЭМ к потребляемой им элек- трической мощности. Холодопроизводительность ТЭМ определяется мощностью ТНЭ, поэтому при заданном режиме тепловыделения она имеет фиксированное значение. В таком случае при определении эффективности охлаждения более информативной характеристикой является величина снижения температуры посадочного места ТНЭ, достигаемая за счет использования ТЭСОТ.

\section{Математическая модель и анализ режимов термоэлектрического охлаждения}

Обычная ТОС имеет в общем виде наиболее простую схему (рис. 1,a). Интегральным параметром, характеризующим эффективность теплопередачи в такой системе, является ее термическое сопротивление $R_{s}$, определяющее величину температурного перепада в зависимости от мощности тепловыделения ТНЭ $Q$

$$
T_{1}-T_{0}=R_{S} Q
$$

Здесь $T_{1}, T_{0}$ - значения температуры посадочного места ТНЭ и окружающей среды.

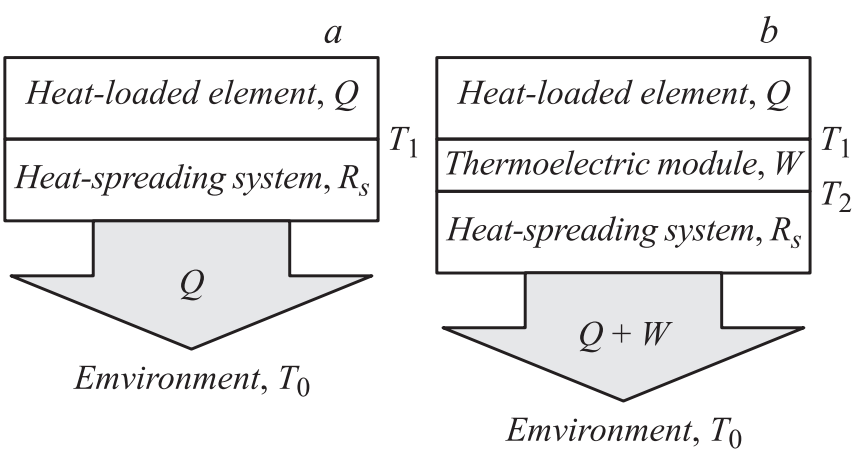

Рис. 1. Схемы обычной теплоотводящей системы $(a)$ и термоэлектрической системы охлаждения и терморегулирования $(b)$. 

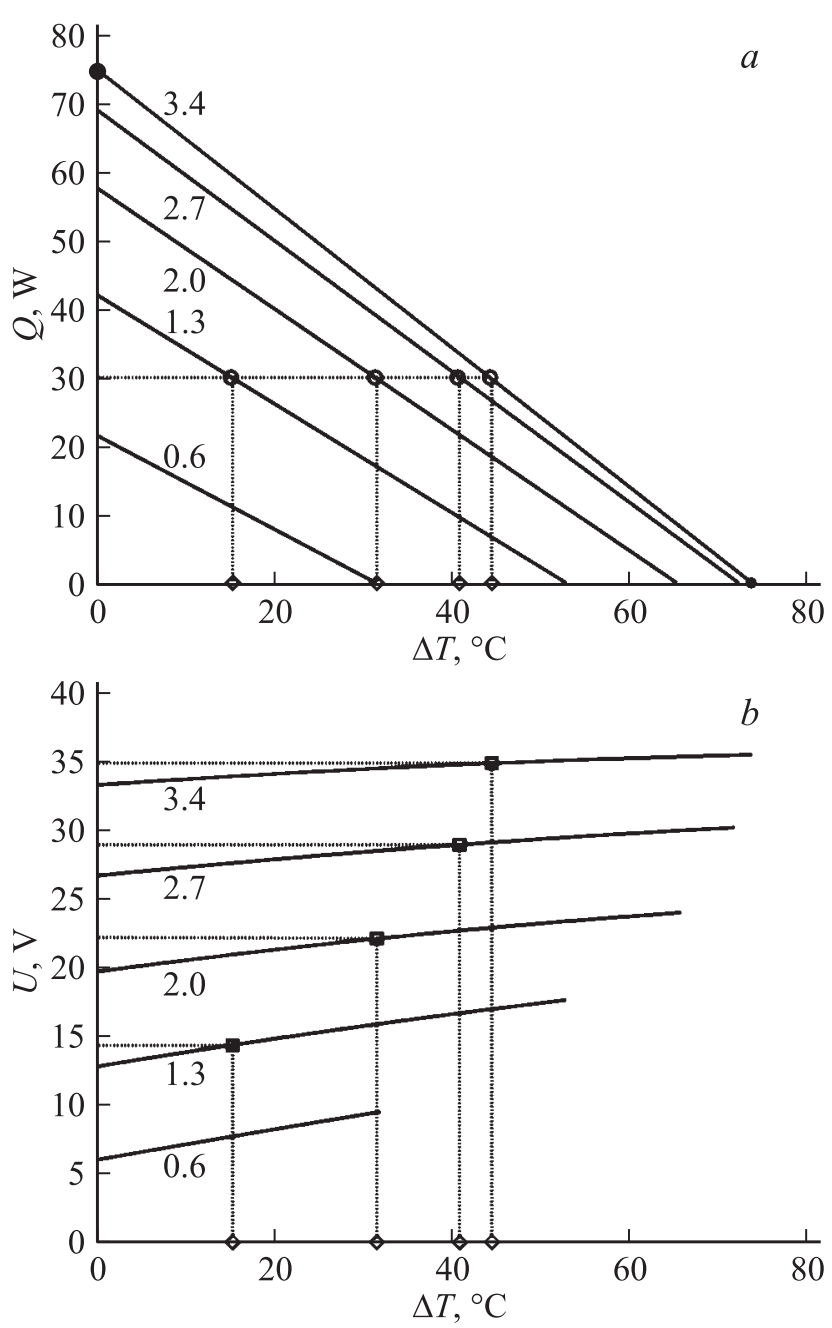

Рис. 2. Зависимости холодопроизводительности $Q\left(\Delta T_{\text {ТЕм }}\right)(a)$ и напряжения $U\left(\Delta T_{\mathrm{TEM}}\right)(b)$ для значений силы тока $I=0.6$, $1.3,2.0,2,7$ и $3.4 \mathrm{~A}$.

В ТЭСОТ между ТНЭ и ТОС дополнительно располагается ТЭМ, потребляющий электрическую мощность $W$ и обеспечивающий перепад температуры $\Delta T_{\mathrm{TEM}}=T_{2}-T_{1}$ между своими сторонами (рис. $\left.1, b\right)$. Для определения температурного перепада в ТЭСОТ воспользуемся аналогичным (1) соотношением

$$
T_{2}-T_{0}=R_{s}(Q+W),
$$

из которого выразим температуру посадочного места ТНЭ

$$
T_{1}=T_{0}+R_{s} Q+R_{s} W-\Delta T_{\mathrm{TEM}}
$$

Формула (2) отличается от выражения $T_{1}=T_{0}+R_{s} Q$, полученного из (1), двумя последними слагаемыми, которые и определяют влияние ТЭМ на температуру посадочного места ТНЭ

$$
\Delta T_{e}=R_{s} W-\Delta T_{\mathrm{TEM}}=R_{s} U(I, Q) I-\Delta T_{\mathrm{TEM}}(I, Q) .
$$

Здесь $U, I-$ напряжение и сила тока электропитания ТЭМ. За счет разницы температуры $\Delta T_{\mathrm{TEM}}$, возника- ющей на противоположных сторонах ТЭМ, обеспечивается охлаждение ТНЭ и, наоборот, к повышению температуры приводит слагаемое $R_{s} W$, ответственное за дополнительный температурный перепад на термическом сопротивлении $R_{s}$ при передаче тепловой мощности $W$, выделяемой ТЭМ. Отрицательное значение $\Delta T_{e}$ соответствует снижению температуры на посадочном месте ТНЭ, при $\Delta T_{e}>0$ температура повышается. При $\Delta T_{e}=0$ температурный режим ТНЭ не меняется, и, следовательно, такой граничный случай соответствует обычной ТОС. Таким образом, величина и знак разности $\Delta T_{e}$ определяют эффективность охлаждения ТЭСОТ.

Как следует из (3), при заданных $R_{s}$ и $Q$ величина $\Delta T_{e}$ зависит от режима работы ТЭМ, а именно от силы питающего тока $I$. При проведении расчетов величины тепловой мощности ТНЭ и термического сопротивления ТОС задавались дискретными значениями $Q=30,40$ и $50 \mathrm{~W}, R_{s}=0.1,0.3$ и $0.5 \mathrm{~K} / \mathrm{W}$ соответственно. Используемые в (3) зависимости $U(I, Q)$ и $\Delta T_{\mathrm{TEM}}(I, Q)$ являются специфическими характеристиками ТЭМ, зависящими от его устройства и используемых материалов ветвей. Такие зависимости можно получить из расчетов $[3,4]$, однако при этом необходимо задать температурные зависимости теплофизических свойств материалов и провести достаточно сложные вычисления. Для серийно выпускаемых ТЭМ наиболее доступной и достоверной информацией по их параметрам и рабочим характеристикам являются данные производителя. При этом наряду с общими параметрами (геометрическими размерами и пр.), как правило, приводятся зависимости $Q(\Delta T)$ и $U(\Delta T)$ для нескольких значений силы тока. На рис. 2 приведены такие зависимости для ТЭМ „РЕ-287-10-15“, которые производятся ОАО „НПП ТФП „ОСТЕРМ СПб“, г. Санкт-Петербург [5].

Рабочие характеристики ТЭМ $Q(\Delta T)$ и $U(\Delta T)$ являются исходными данными для определения зависимостей $U(I, Q)$ и $\Delta T_{\mathrm{TEM}}(I, Q)$ и дальнейшего расчета

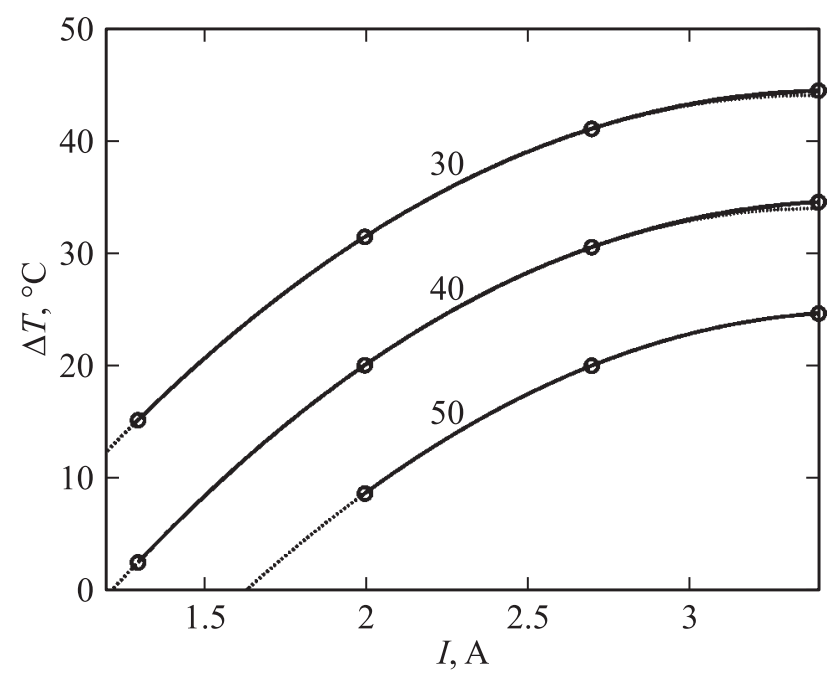

Рис. 3. Зависимости $U(I)$ при $Q=30,40$ и $50 \mathrm{~W}$. 


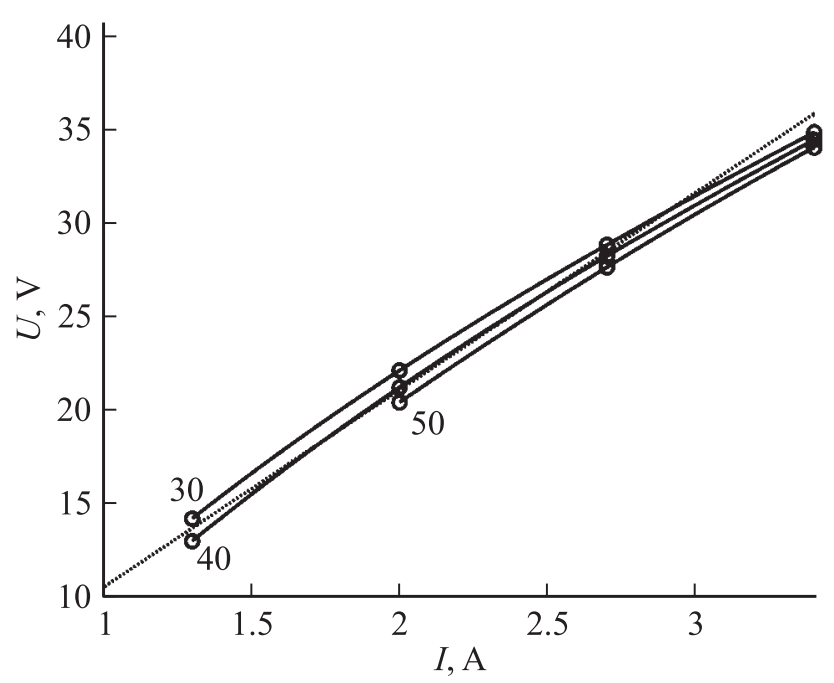

Рис. 4. Зависимости $U(I)$ при $Q=30,40$ и $50 \mathrm{~W}$.

$\Delta T_{e}$. Нагрузочные прямые $Q(\Delta T)$ (рис. 2, $a$ ) отражают обратно пропорциональную зависимость холодопроизводительности $Q$ от температурного перепада $\Delta T$, причем каждая из этих прямых характеризуются двумя значениями $Q_{\max , I}$ при $\Delta T=0$ и $\Delta T_{\max , I}$ при $Q=0$, индекс $I$ указывает на значение силы тока, соответствующее конкретной нагрузочной прямой. В качестве примера на pис. 2, $a$ точками отмечены значения $Q_{\max , 3.4}$ на оси ординат и $\Delta T_{\max , 3.4}$ на оси абсцисс. Для заданного значения холодопроизводительности (на рис. $2, a Q=30 \mathrm{~W}$ ) по нагрузочным прямым несложно определить соответствующие значения $\Delta T_{I}$ для различных значений силы тока

$$
\Delta T_{I}=\left(1-Q / Q_{\max , I}\right) \Delta T_{\max , I}
$$

На оси абсцисс (рис. 2,a) для $Q=30 \mathrm{~W}$ отмечены ромбиками значения $\Delta T_{I}$, с помощью которых по зависимостям $U(\Delta T)$ (рис. 2,b) определяются значения $U_{I}$, отображенные на рисунке квадратиками. На рис. 3 и 4 кружками отмечены значения $\Delta T_{I}$ и $U_{I}$, полученные для $Q=30,40$ и $50 \mathrm{~W}$. По полученным значениям $\Delta T_{I}$ и $U_{I}$ с помощью интерполяционных полиномов построены зависимости $\Delta T(I)$ и $U(I)$, которые на рис. 3 и 4 нанесены сплошными линиями. Для $Q=30$ и $40 \mathrm{~W}$ эти полиномы третьей степени построены по четырем точкам, соответствующим значениям $I=1.3,2.0,2.7$ и 3.4 А. Такая аппроксимация была реализована при проведении компьютерных расчетов эффективности охлаждения ТЭСОТ в [6]. Однако для аналитических расчетов эта методика неприемлема, поскольку применение слишком громоздких соотношений требует непомерно больших вычислительных и временных затрат, а также значительно усложняет анализ полученных соотношений.

В настоящей работе осуществлена адаптация расчетной методики для проведения аналитических вычислений, на основе которых выполнены анализ и оптимизация режимов ТЭСОТ. Для получения более простой зависимости $\Delta T_{\mathrm{TEM}}(I)$ был использован полином второго порядка, который имеет наиболее компактную форму при записи относительно центрального узла

$$
\begin{gathered}
\Delta T_{\mathrm{TEM}}(I)=a\left(I-I_{0}\right)^{2}+b\left(I-I_{0}\right)+c, \\
a=0.5\left(\Delta T_{-1}-2 \Delta T_{0}+\Delta T_{1}\right) / h_{I}^{2}, \\
b=0.5\left(\Delta T_{1}-\Delta T_{-1}\right) / h_{I}, \\
c=\Delta T_{0} .
\end{gathered}
$$

Для значений $Q=30$ и $40 \mathrm{~W}$ полиномы записаны относительно центрального узла при $I_{0}=2 \mathrm{~A}$ и построены по трем последовательным узлам со значениями $\Delta T_{-1}$, $\Delta T_{0}$ и $\Delta T_{1}$, соответствующим значениям силы тока $I=1.3,2.0$ и 2.7 А с шагом $h_{I}=0.7 \mathrm{~A}$. Для $Q=50 \mathrm{~W}$ использованы узлы при значениях силы тока $I=2.0,2.7$ и 3.4 А. Полученные зависимости на рис. 3 нанесены пунктирными линиями. Как видно из рисунка, на базисном интервале, включающем три последовательных узла, пунктирные зависимости практически накладываются на сплошные линии, за пределами интервала появляются незначительные отклонения. Значения интерполяционных коэффициентов для зависимостей $\Delta T_{\mathrm{TEM}}(I)$ при $Q=30$ и $40 \mathrm{~W}$, для которых интерполяция проводилась на одном и том же наборе узлов, соответственно равны $a=-6.8$ и $-7.2, b=18.5$ и $20, c=31.4$ и 20. Различие коэффициентов свидетельствует о том, что кривые отличаются не только уровнем расположения, но также наклоном и формой.

Графики $U(\Delta T)$ (рис. 4) имеют вид, близкий к прямо пропорциональному закону, поэтому в данном случае оправдано использование приближенной зависимости в виде линейной функции

$$
U=R I
$$

где $R$ - электрическое сопротивление ТЭМ, определяемое как отношение соответствующих узловых величин $U_{I}$ к значениям силы тока $I=1.3,2.0$ и $2.7 \mathrm{~A}$. На рис. 4 пунктирной линией для $Q=40 \mathrm{~W}$ приведена линейная зависимость $U(I)$, значение $R=10.53 \Omega$ в которой было определено как средняя арифметическая величина отношения $U_{I} / I$ для трех вышеуказанных узлов. Максимальное отклонение 5\% аппроксимирующей прямой от исходной зависимости $U(I)$ наблюдается на левой границе интервала значений силы тока $1.3-2.7 \mathrm{~A}$, за пределами интервала (при $I=3.4$ A) максимальная погрешность составляет около $4 \%$. При использовании более простого определения $R$, например только по одному среднему узлу, максимальная погрешность аппроксимации увеличивается до $6 \%$. Следует отметить, что по данным производителя электрическое сопротивление ТЭМ, измеряемое при $20^{\circ} \mathrm{C}$, существенно отличается и равно $9.1 \Omega$.

Эффективность охлаждения, количественно выраженная в перепаде температуры $\Delta T_{e}$, рассчитывалась по формуле (3) для заданных значений мощности тепловыделения $Q$ и термического сопротивления $R_{s}$ с учетом 


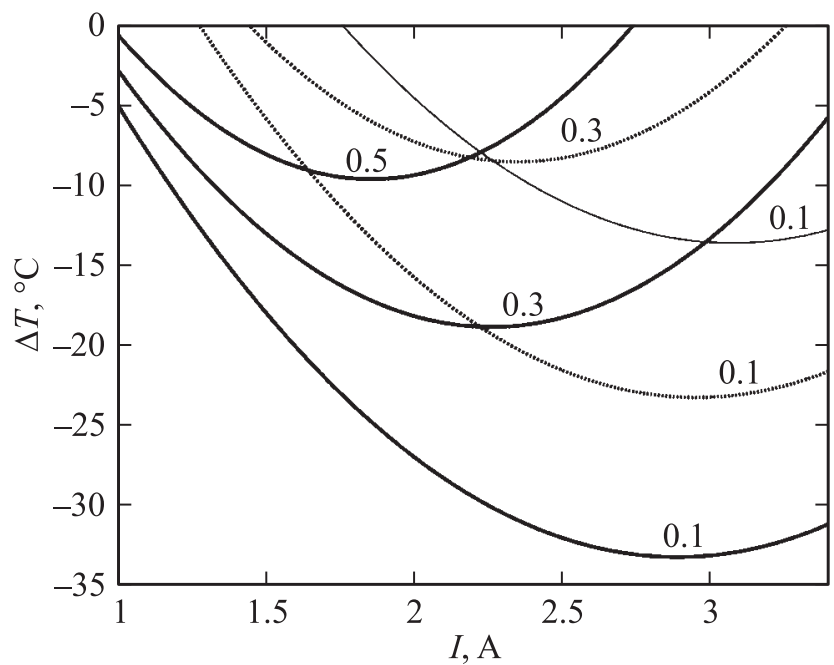

Рис. 5. Зависимости $\Delta T_{e}(I)$ для $Q=30 \mathrm{~W}$ (сплошные жирные линии), $40 \mathrm{~W}$ (пунктирные линии) и $50 \mathrm{~W}$ (сплошная тонкая линия), соответствующие значения $R_{s}$ приведены в $\mathrm{K} / \mathrm{W}$.

зависимостей $\Delta T_{\text {TЕм }}(I)$ и $U(I)$, определяемых соотношениями (4)-(6). На рис. 5 приведены графики $\Delta T_{e}(I)$ для трех значений $R_{s}=0.1,0.3$ и $0.5 \mathrm{~K} / \mathrm{W}$ при $Q=30 \mathrm{~W}$ (сплошные жирные линии), $40 \mathrm{~W}$ (пунктирные линии) и $50 \mathrm{~W}$ (сплошная тонкая линия). Из рис. 5 следует, что величина термического сопротивления оказывает значительное влияние на эффективность охлаждения. Так, минимальные значения $\Delta T_{e}$ при $R_{s}=0.1$ и $0.5 \mathrm{~K} / \mathrm{W}$ отличаются более чем на $20^{\circ} \mathrm{C}$. Минимумы на кривых $\Delta T_{e}(I)$ соответствуют оптимальным режимам с максимальной эффективностью ТЭСОТ, в которых достигается наибольшее снижение температуры. Положение минимумов $\Delta T_{e}$ соответствует оптимальным значениям $I_{\mathrm{opt}, \Delta T}$, которые, как видно из рис. 5, зависят от тепловой мощности ТНЭ и термического сопротивления ТОС. Следует отметить, что для рассматриваемого ТЭМ „РЕ-287-10$15^{\text {“ и }} Q>50 \mathrm{~W}$ возможности снижения температуры ТНЭ даже при минимальном значении $R_{s}=0.1 \mathrm{~K} / \mathrm{W}$ становятся ограниченными $\left(\left|\Delta T_{e}\right|<13^{\circ} \mathrm{C}\right)$, а применение ТЭСОТ малоэффективным.

Значения оптимальной силы тока $I_{\mathrm{opt}, \Delta T}$, при которых достигается максимальное охлаждение, определим по нулевым значениям производной $d\left(\Delta T_{e}\right) / d I$, полученной на основе соотношений (3)-(6),

$$
\frac{d\left(\Delta T_{e}\right)}{d I}=2 R_{s} R I-2 a\left(I-I_{0}\right)-b=0 .
$$

Из (7) выразим величину оптимальной силы тока

$$
I_{\mathrm{opt}, \Delta T}=\frac{b-2 a I_{0}}{2\left(R_{S} R-a\right)} .
$$

Из формулы (8) очевидна обратно пропорциональная зависимость $I_{\mathrm{opt}, \Delta T}$ от $R_{S}$, которая приведена на рис. 6 сплошными линиями для разных значений $Q$. Зависимость $I_{\text {орt, } \Delta T}$ от $Q$ в (8) присутствует в неявном виде через значения интерполяционных коэффициентов $a, b$ и $R$. С ростом величины $R_{s}$ положение минимумов зависимостей $\Delta T_{e}(I)$ смещается в область меньших значений силы тока, для $R_{s}=0.1,0.3$ и $0.5 \mathrm{~K} / \mathrm{W}$ при $Q=30 \mathrm{~W}$ оптимальные значения силы тока соответственно равны $I_{\text {opt }, \Delta T}=2.75,2.1$ и 1.75 A (рис. 5). При этом ТЭСОТ эффективнее обычной системы теплоотвода во всем диапазоне значений $I$, за исключением значений силы тока $I \approx 3.4 \mathrm{~A}$ для $R_{s}=0.5 \mathrm{~K} / \mathrm{W}$.

Термоэлектрические системы охлаждения имеют сравнительно невысокий холодильный коэффициент полезного действия, поэтому при их использовании важной задачей является снижение затрат энергии на охлаждение. В [6] было отмечено, что при определенном уменьшении силы тока питания ТЭМ относительно $I_{\mathrm{opt}, \Delta T}$ обеспечивается существенное падение энергопотребления ТЭМ при незначительном снижении $\Delta T_{e}$. В качестве параметра, характеризующего эффективность режимов термоэлектрического охлаждения с точки зрения минимизации энергетических затрат, введем величину $E$, являющуюся отношением перепада температуры к электрической мощности, потребляемой ТЭМ:

$$
E=\frac{\Delta T_{e}}{W}=R_{s}-\frac{\Delta T_{\mathrm{TEM}}}{W} .
$$

На рис. 7 приведены графики $E(I)$ для трех значений $R_{s}=0.1,0.3$ и $0.5 \mathrm{~K} / \mathrm{W}$ при $Q=30 \mathrm{~W}$ (сплошные жирные линии), $40 \mathrm{~W}$ (пунктирные линии) и $50 \mathrm{~W}$ (сплошная тонкая линия).

Аналогично из уравнения $d E / d I=0$ определим оптимальное значение силы тока для энергетически экономичных режимов охлаждения

$$
I_{\mathrm{opt}, E}=\frac{2\left(b I_{0}-a I_{0}^{2}-c\right)}{b-2 a I_{0}} .
$$

На рис. 6 зависимости $I_{\mathrm{opt}, E}$ отмечены пунктирными линиями. Как следует из формулы (9) и видно из рис. 6

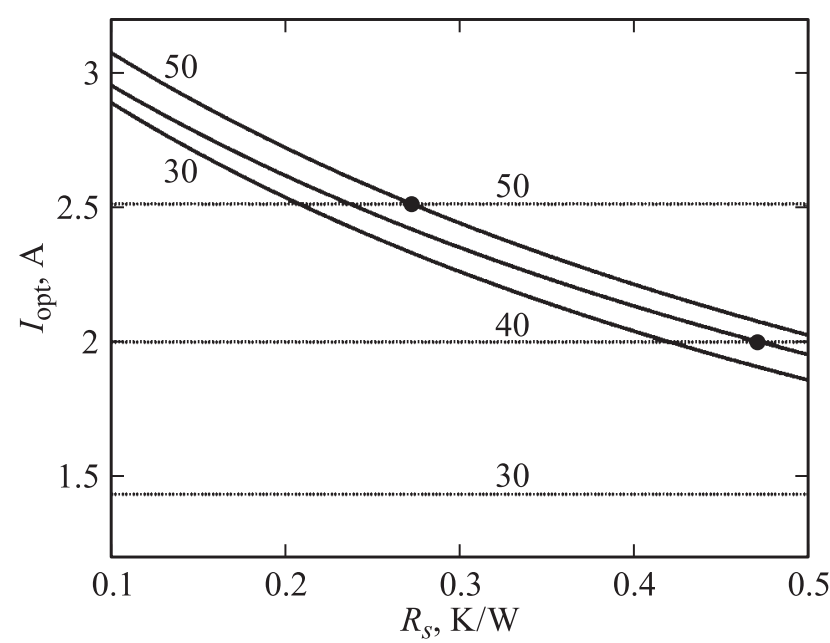

Рис. 6. Зависимости $I_{\mathrm{opt}, \Delta T}\left(R_{S}\right)$ (сплошные линии) и $I_{\mathrm{opt}, E}\left(R_{S}\right)$ (пунктирные линии), соответствующие значения $Q$ приведены в W. 


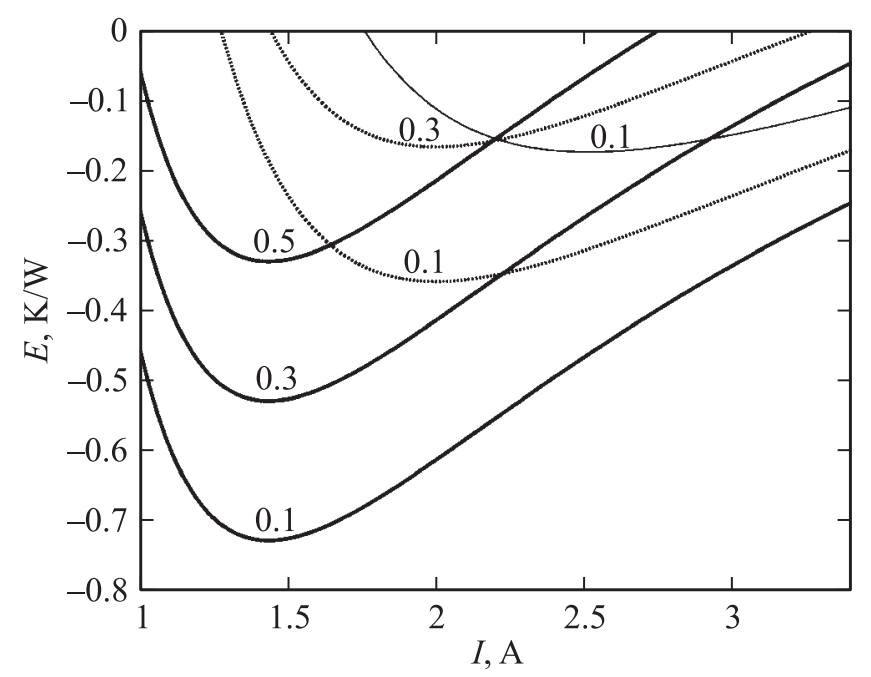

Рис. 7. Зависимости $E(I)$ для $Q=30 \mathrm{~W}$ (сплошные жирные линии), $40 \mathrm{~W}$ (пунктирные линии) и $50 \mathrm{~W}$ (сплошная тонкая линия), соответствующие значения $R_{s}$ приведены в $\mathrm{K} / \mathrm{W}$.

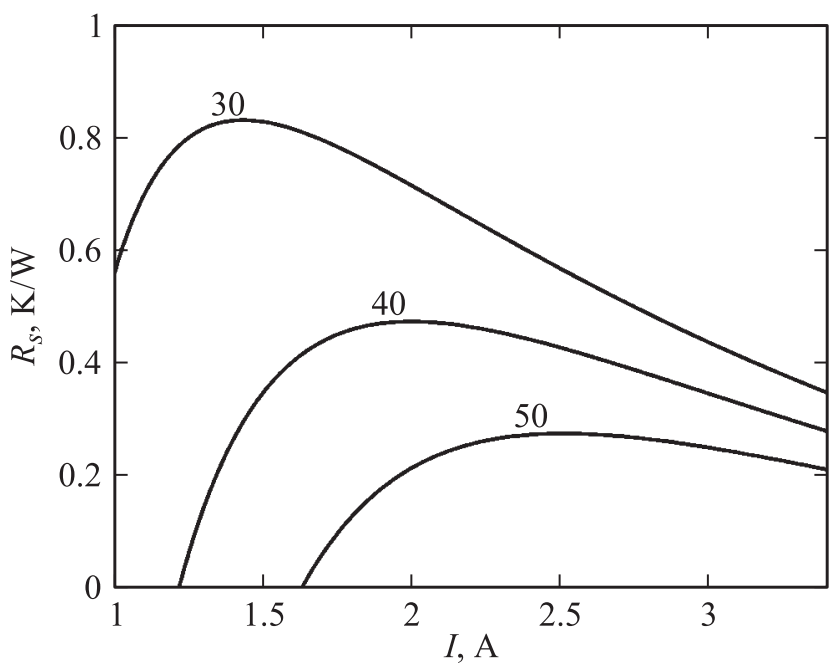

Рис. 8. Границы режимов охлаждения и нагрева ТНЭ, значения $Q$ приведены в W.

и 7, значения $I_{\mathrm{opt}, E}$ не зависят от $R_{s}$. Следует отметить, что в рассматриваемом диапазоне $R_{s}$ зависимости $I_{\mathrm{opt}, E}$ и $I_{\mathrm{opt}, \Delta T}$ имеют общие точки, которые на рис. 6 отмечены для $Q=40$ и $50 \mathrm{~W}$. Действительно, соотношение $d E / d I=0$, записанное в виде

$$
\frac{d E}{d I}=\frac{d\left(\Delta T_{e} / W\right)}{d I}=\frac{\frac{d\left(\Delta T_{e}\right)}{d I} R I^{2}-2 R I \Delta T_{e}}{R^{2} I^{4}}=0,
$$

эквивалентно уравнению

$$
\frac{d\left(\Delta T_{e}\right)}{d I}-\frac{2 \Delta T_{e}}{I}=0
$$

Из (10) следует, что при $\Delta T_{e}=0$ соотношения $d\left(\Delta T_{e}\right) /$ $d I=0$ и $d E / d I=0$ становятся тождественными, при этом графики зависимостей $I_{\mathrm{opt}, \Delta T}$ и $I_{\mathrm{opt}, E}$ пересекаются (рис. 6). Рабочему диапазону ТЭСОТ соответствуют ветви зависимостей, лежащие левее точки пересечения и относящиеся к режимам охлаждения ТНЭ $\left(\Delta T_{e}<0\right)$. Для правых ветвей верно отношение $\Delta T_{e}>0$, следовательно, в этом диапазоне режимов температура ТНЭ будет повышаться.

Как показали приведенные выше результаты расчетов, применение ТЭМ в зависимости от режима его работы, тепловой нагрузки и термического сопротивления ТОС может приводить как к охлаждению ТНЭ, так и к его нагреву. Область рабочих режимов ТЭСОТ, отвечающая условию охлаждения $\Delta T_{e}<0$, определим на плоскости параметров $I-R_{s}$ из уравнения $\Delta T_{e}(I)=0$ с учетом (3) и первого соотношения (5) в виде зависимости $R_{s}(I)$ :

$$
R_{s}=\frac{a\left(I-I_{0}\right)^{2}+b\left(I-I_{0}\right)+c}{R I^{2}} .
$$

Полученные зависимости для $Q=30,40$ и $50 \mathrm{~W}$ приведены на рис. 8. Области рабочих режимов ТЭСОТ расположены ниже соответствующих кривых. При значениях параметров, лежащих в рабочей области, применение ТЭМ обеспечивает дополнительное охлаждение ТНЭ по сравнению с обычной ТОС, за пределами области температура ТНЭ повышается.

\section{Термические сопротивления различных типов TOC}

Анализ эффективности ТЭСОТ был проведен для значений термического сопротивления ТОС $0.1-0.5 \mathrm{~K} / \mathrm{W}$. Оценим возможные диапазоны $R_{s}$ для воздушной, жидкостной и кондуктивной ТОС.

Принудительное воздушное охлаждение осуществляется с помощью воздушных теплообменников с вентилятором (кулеров). Термическое сопротивление современных кулеров для компьютерных процессоров обычно лежит в диапазоне $R_{s}=0.3-0.7 \mathrm{~K} / \mathrm{W}$, лучшие образцы с использованием тепловых трубок достигают $R_{s}=0.1 \mathrm{~K} / \mathrm{W}[7]$.

Жидкостное охлаждение потенциально превосходит воздушное в эффективности, поскольку жидкости имеют значительно бо́льший коэффициент теплообмена с твердой поверхностью, чем газовая среда. В такой ТОС теплота от ТНЭ через теплообменник передается потоку жидкости, движение которой обеспечивается помпой, однако, как правило, отвод теплоты от жидкости в окружающую среду в итоге осуществляется воздушным теплообменником с вентилятором. В таком случае жидкостная ТОС может иметь меньшее общее термическое сопротивление только при условии использования более эффективного воздушного теплообменника. Это условие может быть выполнено за счет того, что воздушный теплообменник не контактирует непосредственно с ТНЭ или ТЭМ, поэтому может располагаться удаленно, имея при этом более развитую поверхность теплообмена и лучшие условия теплообмена с окружающей средой. 

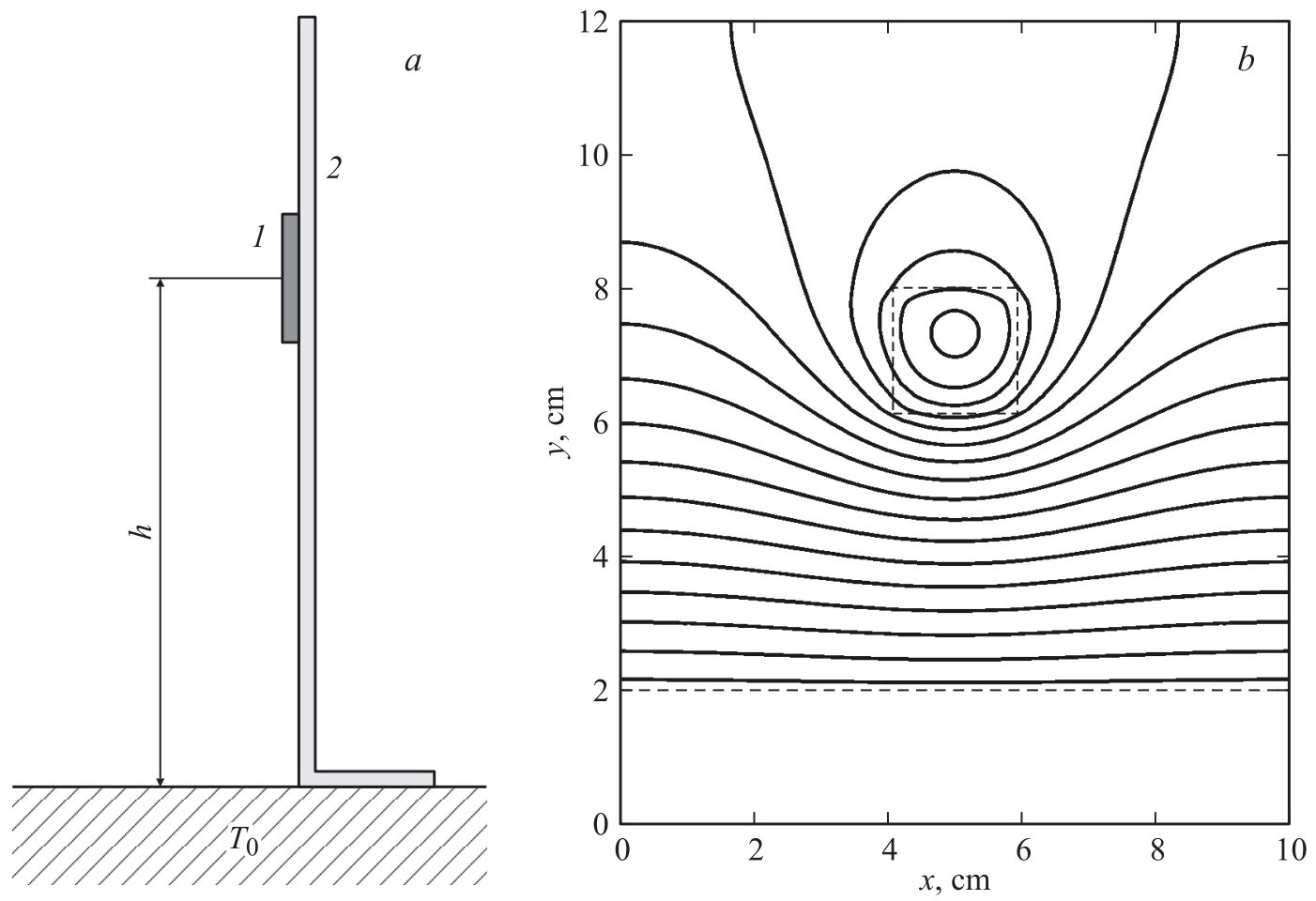

Рис. 9. Конструкция $(a)$ и температурное поле $(b)$ кондуктивной ТОС.

В кондуктивных ТОС одним из самых распространенных вариантов конструкции является крепление ТНЭ на металлическом основании платы или стенке корпуса (рис. 9,a). Термическое сопротивление в такой ТОС определяется конфигурацией системы, теплопроводностью материала, толщиной стенки и другими параметрами. Определим диапазон величины $R_{s}$ для данной системы из расчета теплопередачи только за счет теплопроводного механизма в $L$-образной пластине $(2)$, к которой прикреплен ТНЭ (1) на высоте $h$ (рис. 9, $a$ ). Для этого численно решалось двумерное уравнение теплопроводности, имеющее вид

$$
\lambda\left(\frac{\partial^{2} T}{\partial x^{2}}+\frac{\partial^{2} T}{\partial y^{2}}\right)+q(x, y)-\alpha T=0 .
$$

Здесь $\lambda$ - коэффициент теплопроводности материала пластины, $\alpha-$ коэффициент теплообмена. Два последних слагаемых в (11) описывают боковой теплообмен пластины. В месте крепления ТНЭ задается величина плотности теплового потока $q$, равная отношению тепловой мощности к площади основания ТНЭ. Величина теплового потока, перетекающего между нижней горизонтальной частью (основанием) $L$-образной пластины и ее посадочным местом, определяется выражением

$$
q=\alpha\left(T_{0}-T(x, y)\right) \text {. }
$$

Здесь $T_{0}$ - температура посадочного места пластины.

Расчеты проведены при следующих значениях параметров: размеры вертикальной части пластины со- ставляют $10 \times 10 \mathrm{~cm}$, основания $-10 \times 2 \mathrm{~cm}$, толщина $-1 \mathrm{~mm}$, коэффициент теплопроводности материала $-200 \mathrm{~W} /(\mathrm{m} \cdot \mathrm{K})$, мощность тепловыделения элемента $Q=10 \mathrm{~W}$ на площадке $2 \times 2 \mathrm{~cm}$ соответствует плотности теплового потока $25 \mathrm{~kW} / \mathrm{m}^{2}$, термическое сопротивление теплового стыка между основанием пластины и посадочным местом - $0.03 \mathrm{~K} / \mathrm{W}$, температура посадочного места $T_{0}=20^{\circ} \mathrm{C}$. На рис. $9, b$ приведено распределение температурного поля при значении $h=5 \mathrm{~cm}$, температурный шаг между изолиниями равен $2{ }^{\circ} \mathrm{C}$, штриховыми линиями обозначены габариты посадочной площадки ТНЭ и граница между вертикальной частью и основанием пластины. Максимальное значение температуры под элементом равно $T_{\max }=54.4^{\circ} \mathrm{C}$, в данном случае величина термического сопротивления ТОС $R_{s}=\left(T_{\max }-T_{0}\right) / Q=3.44 \mathrm{~K} / \mathrm{W}$. Очевидно, что увеличение $h$ приводит к повышению $R_{s} \quad\left(R_{s}=5.03 \mathrm{~K} / \mathrm{W}\right.$ при $h=8 \mathrm{~cm})$ и, наоборот, $R_{s}$ снижается при уменьшении $h$ $\left(R_{s}=1.91 \mathrm{~K} / \mathrm{W}\right.$ при $\left.h=2 \mathrm{~cm}\right)$. Изменение коэффициента теплопроводности материала и толщины пластины также приводит к пропорциональному изменению $R_{s}$. Из результатов расчетов следует, что для данного варианта теплоотводящей системы при типичных толщинах пластины $(\approx 1 \mathrm{~mm})$ сложно добиться значений $R_{s}<1 \mathrm{~K} / \mathrm{W}$. Как видно из рис. 8 , для $R_{s}>0.83 \mathrm{~K} / \mathrm{W}$ режимы термоэлектрического охлаждения ТНЭ возможны только при сравнительно малых значениях мощности тепловыделения (менее $30 \mathrm{~W})$.

Использование гипертеплопроводящих панелей (ГТПП) вместо металлических пластин позволяет 
существенно снизить величину $R_{s}$. ГТПП, по сути являющиеся плоскими тепловыми трубами, обладают эффективной теплопроводностью в несколько десятков раз большей, чем теплопроводность алюминия $[8,9]$. При установке радиоэлемента на ГТПП и паянных тепловых контактах возможно снижение суммарной величины $R_{s}$ до значения $0.1 \mathrm{~K} / \mathrm{W}$ и ниже.

\section{Заключение}

Для описания процессов теплообмена в ТЭСОТ представлена аналитическая математическая модель, в которой рабочие характеристики ТЭМ аппроксимированы полиномами первого и второго порядков. На основе модели проведен расчет тепловых режимов ТНЭ для различных значений термического сопротивления ТОС, тепловой мощности ТНЭ и силы тока электропитания ТЭМ. Определены оптимальные значения силы тока, соответствующие как максимальному охлаждению ТНЭ, так и наиболее экономичным режимам. На плоскости $I-R_{s}$ установлены границы режимов охлаждения и нагрева ТНЭ. Проведена сравнительная оценка характерных значений термического сопротивления для воздушной, жидкостной и кондуктивной ТОС.

В работе анализ режимов ТЭСОТ проведен на примеpe ТЭМ „РЕ-287-10-15“, для других ТЭМ с известными рабочими характеристиками он может быть выполнен аналогично.

Исследование выполнено при финансовой поддержке Российского фонда фундаментальных исследований, Правительства Красноярского края, Красноярского краевого фонда поддержки научной и научно-технической деятельности в рамках научного проекта № 16-41-242104.

\section{Список литературы}

[1] Санин В.Н., Андреков И.К., Цапаев А.П., Копылова Н.А. // Известия ВУЗов. Сер. Радиоэлектроника. 2003. № 9. С. 60 65.

[2] Штерн М.Ю., Штерн Ю.И., Шерченков А.А. // Известия вузов. Сер. Электроника. 2011. № 4. С. 30-38.

[3] Марков О.И. // Письма в ЖТФ. 2004. Т. 30. Вып. 13. С. 7-11.

[4] Васильев Е.Н. // Журн. Сибирского фед. ун-та. Сер. Техника и технология. 2015. Т. 8. № 8. С. 1017-1023.

[5] Однокаскадные стандартные ТЭМ. Технические характеристики. Режим доступа: http://www.osterm.ru/products1.html/

[6] Васильев Е.Н. // ЖТФ. 2017. Т. 87. Вып. 1. С. 80-86.

[7] Коновалов Д.А., Лазаренко И.Н., Дроздов И.Г., Шматов Д.П. // Вестник Воронежского. гос. техн. ун-та. 2014. T. 10. № 1. C. 97-104.

[8] Васильев Е.Н., Деревянко В.А., Нестеров Д.А. и др. // Вычислительные технологии. 2009. Т. 14. № 6. С. 19-28.

[9] Васильев Е.Н., Никиборова Е.С. // Вестник Сибир. гос. аэрокосм. ун-та. 2005. Вып. 3. С. 23-26. 